RESEARCH PAPER RP1627

Part of Journal of Research of the National Bureau of Standards, Volume 34, January 1945

\title{
FREQUENCY METER FOR USE WITH GEIGER-MULLLER COUNTER
}

\author{
By Leon F. Curtiss and Burrell W. Brown
}

\section{ABSTRACT}

An improved circuit is described for reading the rate of pulses from a GeigerMüller counter. ${ }^{1}$ Based on the usual procedure of leveling and rectifying the pulses to charge a condenser, the improvements concern a bridge-type vacuumtube voltmeter to read the voltage on the condenser and an arrangement to compensate parasitic potentials developed in the rectifier for the pulses. An adequate source of potentials from one small transformer is described, which renders the circuit useful in portable instuments. Particular care has been taken to design a circuit that is independent of the voltage of the alternating-current mains from which the circuit is operated. A modification of the circuit for rapidly decaying sources is also described.

\section{CONTENTS}

I. Introduction

II. Circuit for gamma-ray exposure meter _.

III. Adjustment of sensitivity .

IV. Circuit for rapidly decaying sources _.

\section{INTRODUCTION}

The use of Geiger-Müller counters can be extended considerably if the accessory equipment for their operation can be made light enough to be readily portable and at the same time thoroughly reliable. Many attempts have been made to achieve this result, but in general the equipment that has been found satisfactory is too heavy, and that which has been sufficiently light is not reliable, since their components are operated beyond their normal capacity. Now that the vaporfilled Geiger-Müller counters have been shown to be quite ${ }^{2}$ trustworthy when simple precautions are taken in preparing them, the way is open for the development of accessory circuits, operated directly from alternating-current mains, which are of moderate weight and in which all parts are operated within their specified rating. There is presented here a description of one such circuit that has given very good performance. Completely assembled in a metal case, the device weighs slightly less than 15 pounds.

L. F. Curtiss, NBS J. Research 23, 479 (1939) RP1246.

2 F. J. Davis and L. F. Curtiss, NBS J. Research 29, 405 (1942) RP1509. 


\section{CIRCUIT FOR GAMMA-RAY EXPOSURE METER}

A diagram of the circuit for operating and reading the output of a Geiger-Müller counter is shown in figure 1. Since one object is to reduce weight, the power transformer selected is the smallest of those made commercially for the operation of radio sets. The secondary, which is center-tapped, delivers a total a-c voltage of about 650 volts and will carry about 40 milliamperes. In addition, it has a 5-volt and a 6.3-volt filament winding. The problem of obtaining about 1,200 volts and 150 volts $\mathrm{d}-\mathrm{c}$ from this transformer is solved by using. one-half the secondary to provide the positive power-supply voltage and the complete secondary, connected to a voltage-doubling circuit

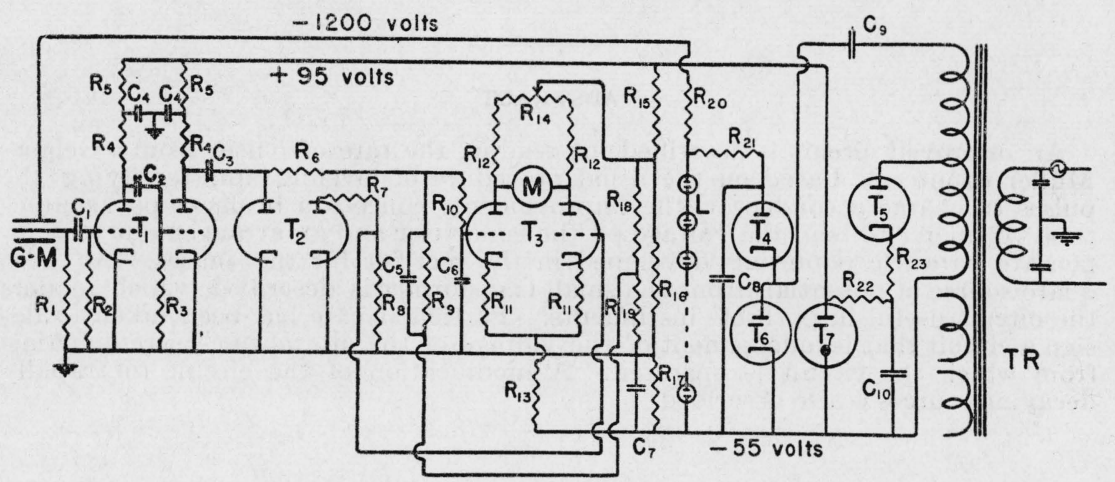

FiguRe 1.-Wiring diagram for gamma-ray exposure meter.

$M=O$ to $200 \mu \mathrm{a} ; L=1 / 10$-watt neon bulbs; $G-M=$ vapor-filled Geiger-Müller counter, $T_{1}=6 \mathrm{SL} 7 ; T_{2}=\mathrm{VR} 150$ $T_{3}=6 \mathrm{~F} 8 ; T_{4}=5 \mathrm{Z} 4 ; T_{5}, T_{6}=6 \mathrm{X} 5 ; T_{7}=\mathrm{VR} 150 ; T R=$ Stancor, P-6010; $C_{1}=100 \mu \mu \mathrm{f}$, mica; $C_{2}=250 \mu \mu \mathrm{f}, \mathrm{mica}$ $C_{3}=60 \mu \mu \mathrm{f}$, mica; $C_{4}, C_{7}=8 \mu \mathrm{f}$, electrolytic; $C_{5}=4 \mu \mathrm{f}$, paper; $C_{6}=0.1 \mu \mathrm{f}$, paper $C_{8}=1 \mu \mathrm{f}, 2000$ volts; $C_{9}=2 \mu \mathrm{f}$, $C_{3}=60 \mu \mu \mathrm{f}, \mathrm{mic} ; C_{4}, C_{7}=8 \mu \mathrm{f}$, electrolytic; $C_{5}=4 \mu \mathrm{f}$, paper; $C_{6}=0.1 \mu \mathrm{f}$, paper $C_{8}=1 \mu \mathrm{f}, 2000$ volts; $C_{9}=2 \mu \mathrm{f}$,
1,000 volts; $C_{10}=16 \mu \mathrm{f}$, electrolytic; $R_{1}=1$ megohm; $R_{2}, R_{3}, R_{4}=0.1$ megohm; $R_{5}=10,000$ ohms; $R_{6}, R_{7}, R_{20}=20$ megohms; $R_{8}, R_{9}=5$ megohms, $R_{10}, R_{21}=3$ megohms; $R_{11}, R_{23}=2,000$ ohms; $R_{12}, R_{15}, R_{16}, R_{17}=20,000$ ohms; $R_{13}=50,000 \mathrm{ohms} ; R_{14}, R_{22}=5,000 \mathrm{ohms} ; R_{18}=100,000 \mathrm{ohms} ; R_{18}=3,500 \mathrm{ohms}$.

as shown, to provide the higher negative voltage. Half-wave rectification is used in both cases. The lower voltage, to supply the plate voltages of the amplifying and indicating circuit, is stabilized by a VR-150 voltage-regulator tube. The higher voltage, to provide the operating potential for the Geiger-Müller counter, is stabilized by a series of $1 / 10$-watt neon lamps that has been thoroughly aged by operation on alternating current for at least 2 weeks. It has been found that lamps so aged maintain the same constant voltage across their terminals for very long periods of time. A voltage-stabilizing circuit connected as shown will show fluctuations unreadable on a 1,500 -volt electrostatic voltmeter when the primary voltage of the transformer is varied from 100 to 130 volts. The actual change may be as much as 5 to 10 volts. This is sufficient regulation for a GeigerMüller tube with reasonably good plateau, and will produce a negligible change in the indication of a good counter.

When using Geiger-Müller counters of the vapor-filled type very little amplification and leveling of pulses is required. Therefore, the relatively simple circuit shown is entirely adequate. The first triode section of $T_{1}$ provides the required amplification of the negative pulse from the counter $G-M$. These pulses are applied to the grid of this triode through a conventional resistance-capacity coupling. The 
pulses at the plate, now positive, are sufficient to saturate the second triode stage, which is operated at zero bias. Therefore, the positive pulses applied to the grid of this triode result in grid rectifications so that the main pulses at the plate of this stage are positive, somewhat broadened, and of equal height and width. As the triode side of $C_{3}$ becomes positive, the diode side accumulates an excess of positive charge which flows through the diode to ground; thus, after the return of the plate of the second half of $T_{1}$ to normal potential, a negative charge is left at the diode plate of $C_{3}$, which flows through $R_{6}$ to the $R C$ circuit, charging it negativ ely. $R_{6}$ serves to prevent the pulses from bypassing the diode by way of $C_{5}$ without rectification. Under these conditions, an equal charge of negative sign is applied to the condenser, $C_{5}$, for each pulse. Since condenser $C_{5}$ has a resistor, $R_{9}$, connected across it, the potential across the condenser will rise to a constant value for a constant rate of pulses from the Geiger-Müller counter.

Therefore, to obtain a reading proportional to the rate of pulses at the Geiger-Müller counter, a reliable electronic voltmeter is required to measure the voltage across condenser $C_{5}$. This is provided by the twin-triode $T_{3}$, with the two sections connected in a bridge circuit with a microammeter, $M$, connected between the plate terminals of the two sections. These triodes are operated at a negative bias provided through high-resistance cathode resistors $R_{13}$ and $R_{11}$ in such a way that the readings of the meter are fairly independent of the characteristics of the triode sections. A balancing potentiometer, $R_{14}$, is provided to adjust the circuit for the residual effects of differences between the triode sections. This adjustment is made with the Geiger-Müller counter disconnected. In general, it need only be changed when replacing a tube.

To balance out certain parasitic potentials arising from the use of a diode to rectify the pulses from the counter, the two grids of the twin triode, $T_{3}$, are connected in identical circuits, each to one section of the twin diode, $T_{2}$. In addition, it is desirable to compensate the blank counting rate of larger Geiger-Müller tubes so that the meter reads zero in the absence of a source of radiation. This is done by applying a slight positive bias to the grid of the voltmeter tube attached to the condenser, $C_{5}$, by a bleeder circuit composed of resistors $R_{18}$ and $R_{19}$. This adjustment can be made by proper selection of $R_{18}$ and $R_{19}$ for a given Geiger-Müller tube and need be changed only when replacing this tube.

The resultant circuit has the following desirable characteristics:

1. The reading of the meter, $M$, is quite accurately proportional to the rate of pulses from the counter. The calibration of intensity of radiation versus the reading of the meter is a straight line passing through the origin in a correctly adjusted circuit. This is shown in figure 2, which is a typical calibration for one of these circuits.

2. The reading of the meter is practically independent of the $a-c$ supply voltage in the range from 100 to 130 volts. This 30 -volt change produces less than a 2 percent change in the reading of the meter.

3. No component is operated above its normal rating, so that failure of vacuum tubes, condensers, and resistors is reduced to a minimum.

4. All potentials, both positive and negative, are obtained from one transformer, thus reducing the weight and with no sacrifice in performance. 


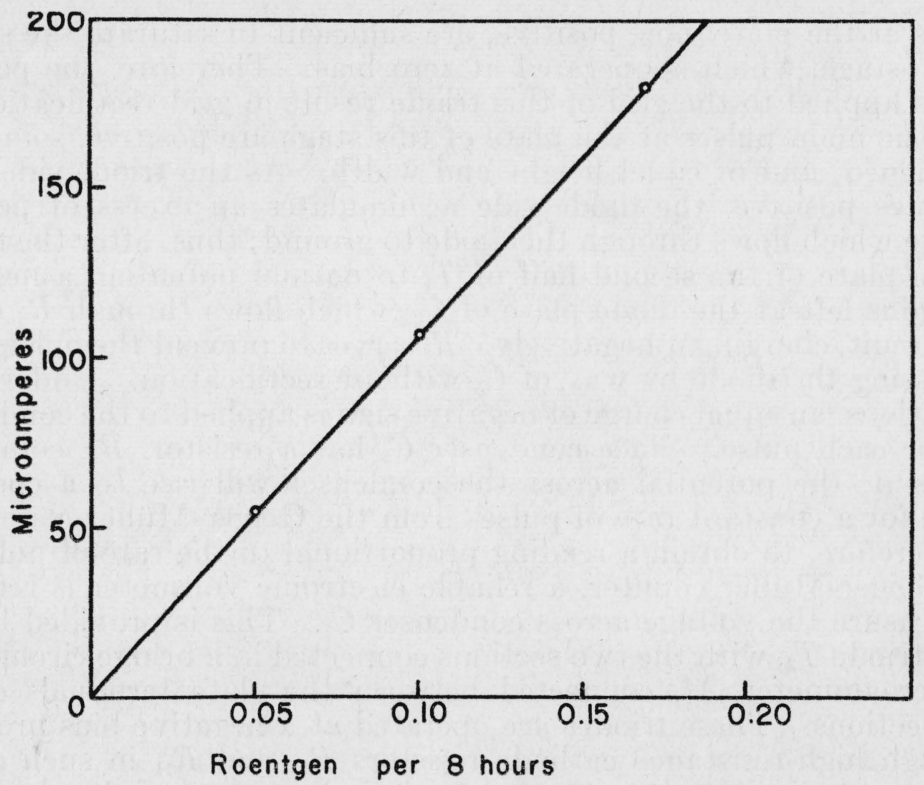

Figure 2.-Calibration curve for the gamma-ray exposure meter.

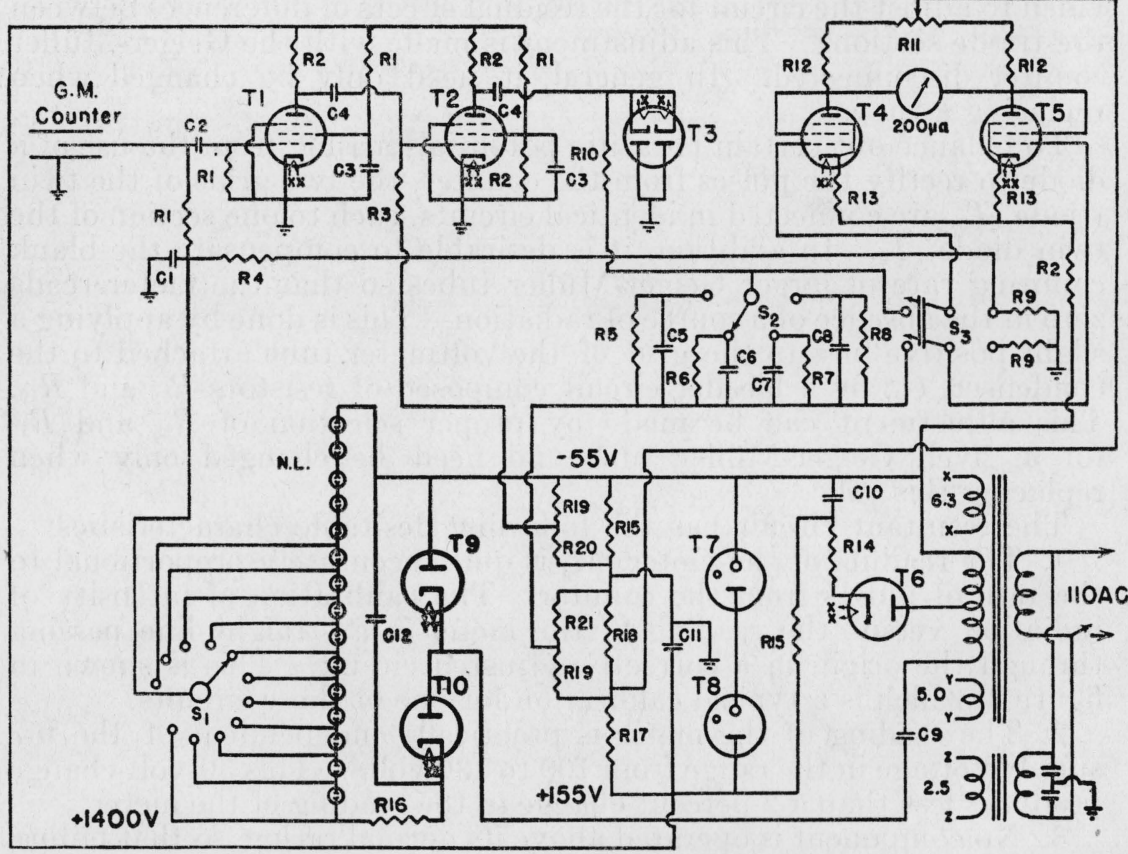

FIGURE 3.-Wiring diagram for circuit for rapidly decaying sources.

$T_{1}, T_{2}=6 \mathrm{AC} 7 ; T_{3}=6 \mathrm{H} 6 ; T_{4}, T_{5}=6 \mathrm{~K} 6 ; T_{6}=6 \mathrm{X} 5 ; T_{7}, T_{8}=\mathrm{VR} 105 ; T_{9}=5 \mathrm{Z} 4 ; T_{10}=2 \mathrm{X} 2 ; C_{1}=0.02 \mu \mathrm{f} ; C_{2}=50 \mu \mu \mathrm{f}$ 2,500 volts; $C_{3}=0.1 \mu \mathrm{f} ; C_{4}=50 \mu \mu \mathrm{f} ; C_{5}=4 \mu \mathrm{f} ; C_{6}=2 \mu \mathrm{f} ; C_{7}=1 \mu \mathrm{f} ; C_{8}=0.5 \mu \mathrm{f} ; C_{9}=1 \mu \mathrm{f}, 1,000$ volts; $C_{10}=20 \mu \mathrm{f} ;$ $C_{11}=8 \mu \mathrm{f} ; C_{12}=1 \mu \mathrm{f}, 2,000$ volts; $R_{1}=0.1$ megohm; $R_{2}=50,000$ ohms; $R_{3}, R_{4}=60$ megohms; $R_{5}, R_{10}=0.2 \mathrm{meg}$ ohm; $R_{6}=0.67$ megohm; $R_{7}=2$ megohms; $R_{8}=6$ megohms; $R_{9}=60,000$ ohms; $R_{11}=5,000$ ohms; $R_{19}, R_{13}, R_{20}=$ 20,000 ohms; $R_{14}=1,000$ ohms; $R_{15}=5,000$ ohms; $R_{16}=2$ megohms; $R_{17}=25,000$ ohms; $R_{18}=10,000$ ohms; $R_{19}=100,000$ ohms. 


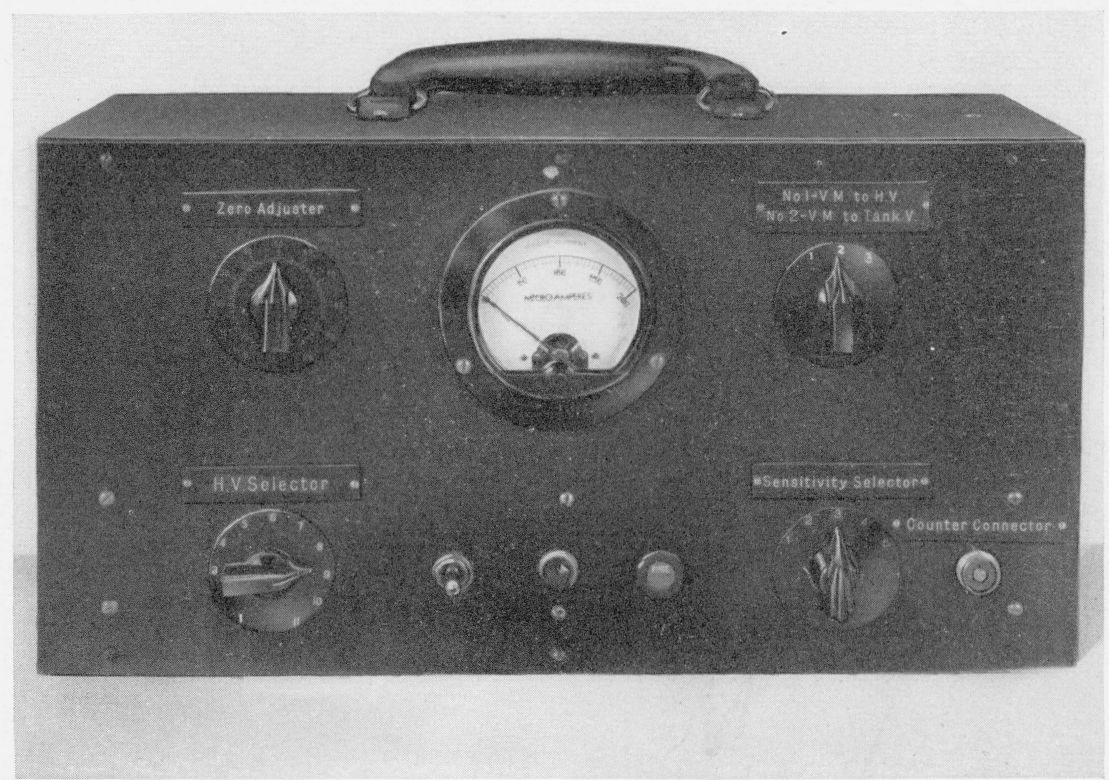

Figure 4.-View of panel of counting-rate meter for use with Geiger-Müller counter. 


\section{ADJUSTMENT OF SENSITIVITY}

Since the first twin triode operates as a unit to provide the required pulses for rectification, the only circuit change required to alter the reading of the meter, $M$, for a given pulse rate, is that of changing the capacitance of $C_{3}$. Increasing this value for $C_{3}$ will increase the quantity of charge per pulse applied to $C_{5}$. This means of adjustment is adequate for such purposes as to adjust the meter reading so that a given intensity will come at an appropriate scale division. Large changes in sensitivity are best obtained by changing the size of the Geiger-Müller counter tube. The range of intensity that can be measured with a given counter will usually extend to about 10 times the lowest value for which the counter will give a satisfactory reading. Above this value the rate of pulses is so high that the "dead time" destroys the linearity of response. Therefore, the large counters useful for the measurement of low intensities fail to operate satisfactorily when the intensity rises above a certain limit. The remedy is to use a smaller counter tube for the higher intensities. The circuit described here works equally well, with minor modifications, for Geiger-Müller tube counters ranging in size from 0.5 by $1.0 \mathrm{~cm}$ to 5 by $30 \mathrm{~cm}$, according to our experience. The calibration curveshown in figure 2 is for a counter tube 1 by $1 \mathrm{~cm}$ as a gamma-ray dosage indicator for an 8-hour exposure in terms of roentgens.

\section{CIRCUIT FOR RAPIDLY DECAYING SOURCES}

In the adaptation of the preceding circuit to the use of GeigerMüller counters for rapidly decaying sources and for use with activated foils in direct contact with the counter, considerable changes are necessary. The high-voltage polarity must be reversed and applied to the central electrode through a resistor, so that the outer electrode may be operated at ground potential. To allow flexibility in use and simplicity in replacement of faulty or badly contaminated counters, a 3-foot single conductor, low-capacity, shielded cable and one amphenol cable connector are used to connect the counter to the amplifier. For simplicity in connecting the counter to the cable, a short section of the high-voltage wire and counter terminal were left unshielded, with no apparent trouble from pick-up of stray electrical disturbances. The high-voltage selector, $S_{1}$, permits counters with widely different working voltages to be used with a minimum of trouble in going from one to the other. A switch and voltage divider system is provided for connecting the voltmeter circuit to the high voltage for rapid checks.

Since linear response to counting rates up to 100,000 per minute is desirable, $6 \mathrm{AC} 7$ tubes are substituted as amplifiers, and the time constants are kept fairly small. Referring to figure 3 , it is to be noted that the screen-grid voltages on $T_{1}$ and $T_{2}$ are supplied by different methods, $T_{1}$ by a dropping resistor, and $T_{2}$ by a voltage divider. Since more than enough signal is available at the grid of $T_{2}$ to drive it to saturation, the grid is biased beyond the cutoff point, which reduces the tendency to oscillate, and therefore eliminates the necessity for decoupling resistors and capacitors in the plate circuit, and removes small pulses and ripples due to leakages and imperfect filtering in the voltage supplies. 
In this circuit, the pulse arriving at rectifier $T_{3}$ is negative; therefore, to get rectification the plates are tied together and grounded and the signal is applied to the cathode. Since both d-c voltage supplies are regulated, variations caused by line-voltage fluctuations must be due to changes in heater voltage. In the previous circuit, these were balanced out by attaching the voltmeter terminals to symmetrical points with reference to the two halves of the $6 \mathrm{H} 6$. Here it was found that at normal heater voltage there exists a potential of about 0.8 volt between cathode and plate, which is dependent upon the heater voltage. ${ }^{3}$ If, however, the cathode is made about 1 -volt positive with respect to the plate, the line voltage is not likely to vary enough to cause this parasitic potential ever to rise beyond this value. Therefore, the voltage across the $R C$ circuit is dependent only on the number of pulses arriving at the rectifier and the constants chosen for this circuit. $S_{2}$ is a four-point rotary switch, which offers sensitivity ranges varying from approximately 3,000 to 100,000 pulses per minute for maximum deflection of the meter.

An added advantage in this circuit is that the resistor $R_{10}$ between the rectifier and $R C$ circuit can be as low as $0.1 \mathrm{megohm}$ and still perform quite satisfactorily. In the previous circuit, however, this resistor was kept larger to minimize the effect of this parasitic potential on the linearity of the $R C$ circuit. The smaller the total resistance from the cathode of the rectifier to ground, the higher the number of pulses per unit time to which the tank circuit will respond linearly. If, however, $R_{10}$ is made too low, the net charge delivered to the tank circuit per pulse becomes too small to give adequate voltage with reasonable values of $R$ in the $R C$ circuit.

In instruments where portability and size are important factors, the twin triode is adequate for the bridge-type vacuum-tube voltmeter, but a little better performance is obtained if $6 \mathrm{~K} 6$ tubes are used instead. The reversal of the polarity of the high voltage is, however, responsible for the greatest change in size and weight. A single power transformer is no longer adequate for the entire voltage supply, because the ordinary commercial power transformer does not have sufficient insulation between its windings to withstand 1,500 to 1,800 volts, which is necessary in this circuit. Since a separate transformer is necessary to heat the cathode of $T_{10}$, one was chosen that would give 2.5 volts, so that a $2 \mathrm{X} 2$ high-vacuum, high-voltage rectifier could be used. The panel of a completed instrument is shown in figure 4. This model as shown weighs slightly less than 20 pounds.

\footnotetext{
941).

3 J. Millman and S. Seely, Electronics, p. 204, ed. 1 (MeGraw-Hill Book Co., Inc., New York, N. Y
}

Washington, August 7, 1944. 